Research Article

\title{
Differences in the Cardiovascular Risk Assessment in Cardiology Outpatients in Mali: Comparison between Framingham Body Mass Index-Based Tool and Low-Information World Health Organization Chart
}

\author{
Bâ Hamidou Oumar $\mathbb{D}^{1},{ }^{1}$ Sangaré Ibrahima, ${ }^{1}$ Camara Youssouf, ${ }^{2}$ Sidibé Noumou, \\ Coulibaly Souleymane, ${ }^{3}$ Cissoko Yacouba, ${ }^{3}$ Sidibé Samba, ${ }^{3}$ Konaté Massama, ${ }^{4}$ \\ Maiga Asmaou Kéita, ${ }^{5}$ Doumbia Coumba Thiam, ${ }^{2}$ Touré Mamadou, ${ }^{1}$ Diakité Mamadou, ${ }^{3}$ \\ Menta Ichaka, ${ }^{1}$ and Diall Ilo Bella ${ }^{3}$ \\ ${ }^{1}$ University Hospital Gabriel Touré, Cardiology Bamako (Mali), Bamako, Mali \\ ${ }^{2}$ University Hospital Bocar Sidy Sall, Cardiology Kati (Mali), Bamako, Mali \\ ${ }^{3}$ University Hospital Point G, Cardiology Bamako (Mali), Infectious Diseases Bamako (Mali), Bamako, Mali \\ ${ }^{4}$ University Hospital "Hôpital Du Mali", Cardiology Bamako (Mali), Bamako, Mali \\ ${ }^{5}$ Mother-Children University Hospital "Le Luxembourg”, Cardiology Bamako (Mali), Bamako, Mali \\ Correspondence should be addressed to Bâ Hamidou Oumar; bhamiba@yahoo.fr
}

Received 1 October 2020; Revised 20 December 2020; Accepted 6 February 2021; Published 28 February 2021

Academic Editor: Kai Hu

Copyright (c) 2021 Bâ Hamidou Oumar et al. This is an open access article distributed under the Creative Commons Attribution License, which permits unrestricted use, distribution, and reproduction in any medium, provided the original work is properly cited.

Objective. This study aimed to compare 2 laborless tools, namely, the body mass index-based Framingham (bmi-Frm) and lowinformation WHO- (li-WHO-) based risk scores, and assess their agreement in outpatients in a cardiology department. Methodology. Data stem from a cross-sectional previous study performed from May to September 2016 in the Cardiology Department of University Hospital Gabriel Touré (UH-GT) in Bamako. All patients aged 40 and more were included in the study allowing the assessment of bmi-Frm and li-WHO prediction charts. The cardiovascular risk (CVR) was evaluated using a calculator prepared by D'Agostino et al. for the bmi-Frm and the li-WHO chart for the Afro-D region of the WHO. The risk score for both ranged from $<10$ to $\geq 40$. The data were entered in an ACCESS 2010 database, then processed by MS Excel 2010, and finally analysed using IBM SPSS Statistics 20. Continuous variables were presented as means and standard deviations, and categorical variables were presented as frequencies with percentages. $P<0.05$ was considered the statistical significance level. After sample description, the risk score was assessed using bmi-Frm and li-WHO prediction tools. Finally, a kappa test was performed to check for the interreliability of both methods. For weighted kappa, coefficients were given all five classes of risk groups in 0, 25 steps from 1 for total concordance to 0 for total discordance. Results. This study involved 793 outpatients, $63.7 \%$ being female, $35.1 \%$ of them younger than 50 years, $57.9 \%$ with no formal education, and $67.7 \%$ with no medical insurance. Means for age, body mass index (BMI), and systolic blood pressure (SBP) were, respectively, $53.81 \pm 16.729$ years, $25.29 \pm 06.151 \mathrm{~kg} / \mathrm{m} 2$, and $139.49 \pm 27.110 \mathrm{~mm}$ Hg. Using the li-WHO prediction chart gives a much higher proportion of low-risk patients compared to bmi-Frm (83.6 vs. 37.7). Sociodemographic characteristics such as education or income level were not different in risk score neither for the bmi-Frm nor for the li-WHO risk score. The percentage of agreement between both tools was $40.4 \%$, and agreement (kappa of 0.1 and weighted kappa of 0.2) was found to be slight. Conclusion. Using the bmi-Frm and li-WHO tool gives a similar risk estimation in younger female patients. Older patients must be evaluated using high-information tools with cholesterol, e.g., versions of the Framingham risk equation or WHO using cholesterol. These must be confirmed in further studies and compared to data from prospective studies 


\section{Introduction}

Cardiovascular diseases (CVD) are the number one cause of death worldwide with 17.9 million deaths in 2016 and made $31 \%$ of all causes of death mainly in low- and middle-income countries (LMIC) [1]. Furthermore, highincome countries (HIC) experience a heavy burden of CVD [2] and therefore underlying cardiovascular risk factors. CVD remain a major cause of health loss in all regions of the world [3]. Actions are therefore necessary and some of them easy to implement [4], already in childhood [5] and through implementing cost-effective population-wide strategies [6].

In Africa, particularly in sub-Saharan countries like most LMIC, there is a shift with regression of infectious diseases and increase of noncommunicable diseases [7]. Our country like most LMIC has a high burden of cardiovascular diseases due to the high prevalence of cardiovascular risk factors [8-12], low-level knowledge and awareness, and also a perception of cardiovascular diseases and their risk factors [13].

As treatment cost remains unaffordable for most people in LMIC, prevention is particularly important for chronic diseases such CVD. WHO-PEN strategy adopted by many countries and intervention trials could lead to a decrease of the burden of CVD [14-16].

To allow the prevention of CVD, tools for risk score assessment have been developed for decades, and most of them for HIC [17] and with challenges [18]. One of the most known is the Framingham risk score (FRS) which has the advantage of a published equation allowing an automatic calculation. Only one tool can be considered worldwide, namely, the prediction tool provided by the World Organization of Health and International Society of Hypertension (WHO/ISH) for all WHO 14 subregions [19].

Opposed to the WHO/ISH prediction chart [20], FRS has been used for various populations [21-24].

Since laboratory tests are expensive, laborless tools such as these 2 cited above are the main tools which can be easily used for the prevention and management of patients with cardiovascular diseases and risk factors. Despite the fact that these tools are essential, data on their use and results are scarce.

To fill this lack of publication and guide their use, this study was performed, aiming to compare 2 laborless tools, namely, the body mass index-based Framingham (bmi-Frm) and low-information WHO- (li-WHO-) based risk scores, and assess their agreement in outpatients in a cardiology department.

\section{Methods}

Data for this study stem from a cross-sectional one carried out from May to September 2016 in the Cardiology Department of University Hospital Gabriel Touré (UH-GT) in
Bamako. We selected patients aged 40 and more with available data allowing FRS estimating.

2.1. Sampling and Data Collection. We included all patients who came to visit during the study time since the required data are already part of the usual clinical exam.

A minimal size of 384 patients was needed for the study using the following formula: $\mathbf{n}=\mathbf{z}^{2} \times \mathbf{p}(\mathbf{1}-\mathbf{p}) / \mathbf{m}^{2}$. This was obtained with a $P$ value set at 0.5 , a confidence interval of $95 \%, z$ of 1.96 , and a margin error of $5 \%$.

Briefly, all patients underwent history taking and complete clinical examination with measures of weight, height, blood pressure, waist, and hip circumference.

Data were first collected using a questionnaire and then inserted in a MS Access database built for this purpose.

\subsection{Definition of Terms}

(i) Height and weight were measured using a rigid stadiometer and digital balance, respectively. Readings were recorded to the nearest centimetre and the nearest $\mathrm{kg}$, with participants being weighted and measured without shoes and only light clothed.

(ii) Body mass index (BMI) was derived from weight and height as weight $(\mathrm{kg}) /$ height $^{2}\left(\mathrm{~m}^{2}\right)$

(iii) Hip circumference (HC) was measured around the widest portion of the buttock whereas waist circumference (WC) was measured with a nonstretchable tape over the light-clothed abdomen at the narrowest point between the lowest rib and the iliac crest. The waist-to-hip ratio (WHr) was WC divided by $\mathrm{HC}$.

(iv) Income level was based on a multiple of the minimum obligatory salary (MOS) (actually 40000 FCFA or 71, 5 USD) from 1 to 4 MOS.

(v) Education level was assessed in terms of years attending in school with four levels defined as follows:

(vi) None: no school attending

(vii) Primary school: 1 to 6 years

(viii) High school: 7 to 12 years

(ix) University: 12 years or more

(x) Blood pressure has been measured using a CONTEC 08A electronic sphygmomanometer allowing the assessment of mean arterial pressure from systolic blood pressure (SBP) and diastolic blood pressure (DBP) by adding to the DBP a third of the differential pressure.

2.3. Risk Assessment. The cardiovascular risk (CVR) was evaluated using a calculator prepared by D`Agostino et al. [15] and the cholesterol-free WHO prediction chart for the Afro$\mathrm{D}$ region. For both, risk assessment was from $<10$ to $\geq 40$. 
2.4. Data Analysis. The data were first exported from the ACCESS database to MS Excel and finally analysed using IBM SPSS Statistics 20. Continuous variables are presented as means and standard deviations, and categorical variables are presented as frequencies with percentages. $P<0.05$ was considered the statistical significance level.

After sample description, the risk score was assessed using bmi-Frm and li-WHO prediction tools. Finally, a kappa test was performed to check for the interreliability of both methods. For weighted kappa, coefficients were given all five classes of risk groups in 0, 25 steps from 1 for total concordance to 0 for total discordance.

2.5. Ethical Approval. The study as part of a thesis for the medical student has been approved by the institutional board of the UH-GT.

2.6. Results. The study involved 793 outpatients, $63.7 \%$ (505) being female and $35.1 \%$ (278) younger than 50 years. Most patients had no formal education (57.9\%) and no medical insurance (67.7\%). More than half of the patients did $\mathrm{n}$ give an income level, and the remaining $12.9 \%$ and $13.9 \%$ had income levels of 1 and 4, respectively (Table 1).

Using both tools, patients with a risk $<10$ were highly represented in different proportions with $83.5 \%$ for li-WHO and $37.7 \%$ for bmi-Frm prediction (Figure 1).

\section{Sociodemographic Characteristics and Risk Scores (Table 3)}

3.1. With bmi-Frm Tool. Patients without medical insurance had $43.2 \%$ versus $26.2 \%$ for those with medical insurance in the lowest risk group and $15.5 \%$ versus $21.9 \%$ in the highest risk group. The proportion of patients decreased from $<10$ to $30-<40$ and increased in the highest risk score $(\geq 40)$ for both patients with and without medical insurance $(P<0.001)$.

Female patients had $49.1 \%$ in the lowest group and 7, 9\% in the highest versus, respectively, $17.7 \%$ and $34.4 \%$ for male patients $(P<0.001)$.

There was a clear trend in the lowest and highest age groups with a decrease of the proportion of patients from $86.0 \%(<10)$ to $0.0 \%(\geq 40)$ for the age group $40-49$ and opposite behaviour for patients $\geq 70$ with $1.4 \%(<10)$ to $17.5 \%(\geq 40)(P<0.001)$.

Education level and income level showed no statistical difference according to the risk score with respective $P$ values of 0.944 and 0.175 .

3.2. With li-WHO Tool. Female and male patients showed the highest proportion of risk group $<10$ with, respectively, $86.7 \%$ and $78.7 \%$.

The low-risk group $<10$ was the most important but in decreasing proportions with increasing age.

As for bmi-Frm, education and income level showed no statistical difference according to the risk score ( $P$ values of 0.624 and 0.569 , respectively).
TABLE 1: Sociodemographic characteristics of the sample of 793 patients in the CVR assessment study.

\begin{tabular}{lccc}
\hline Variables & & $N$ & $\%$ \\
\hline \multirow{2}{*}{ Sex } & $\mathrm{M}$ & 288 & 36.3 \\
& $\mathrm{~F}$ & 505 & 63.7 \\
\hline \multirow{3}{*}{ Age group (years) } & $<40-49$ & 278 & 35.1 \\
& $50-59$ & 169 & 21.3 \\
& $60-69$ & 203 & 25.6 \\
& $\geq 70$ & 143 & 18.0 \\
\multirow{3}{*}{ Education level (school years) } & None & 459 & 57.9 \\
& Primary & 157 & 19.8 \\
& High school & 111 & 14.0 \\
Medical insurance & University & 066 & 08.3 \\
\hline \multirow{2}{*}{ Income level (in multiple of MOS ${ }^{*}$ ) } & No & 537 & 67.7 \\
& Yes & 256 & 32.3 \\
\hline & NA & 435 & 54.9 \\
& 1 & 102 & 12.9 \\
& 2 & 057 & 07.2 \\
& 3 & 089 & 11.2 \\
& 4 & 110 & 13.9 \\
\hline
\end{tabular}

${ }^{*}$ Minimal obligatory salary. Means for age, BMI, and SBP were, respectively, $53.81 \pm 16.729$ years, $25.29 \pm 06.151 \mathrm{~kg} / \mathrm{m} 2$, and $139.49 \pm 27.110 \mathrm{~mm} \mathrm{Hg}$ (Table 2).

TABLE 2: Means for anthropometric and pressure values in the sample of 793 patients in the CVR assessment study.

\begin{tabular}{|c|c|c|c|}
\hline & Mean & Min-max & SD \\
\hline Age (years) & 53.81 & $16-96$ & 16.729 \\
\hline Weight (kg) & 68.43 & $29-137$ & 16.564 \\
\hline Height $(\mathrm{cm})$ & 164.60 & $143-187$ & 08.057 \\
\hline $\mathrm{BMI}^{*}\left(\mathrm{~kg} / \mathrm{m}^{2}\right)$ & 25.29 & $11.9-50.3$ & 06.151 \\
\hline $\mathrm{WC}^{* *} \mathrm{~cm}(775)$ & 88.49 & $50-138$ & 14.024 \\
\hline $\mathrm{HC}^{* * *} \mathrm{~cm}(633)$ & 96.17 & $55-190$ & 14.049 \\
\hline $\mathrm{WHR}^{* * * *}$ (632) & 0.92 & $0.47-1-23$ & 0.069 \\
\hline $\mathrm{HR}+/ \min (685)$ & 83.24 & $43-180$ & 16.067 \\
\hline $\mathrm{SBP}++\mathrm{mm} \mathrm{Hg}$ & 139.49 & $76-230$ & 27.110 \\
\hline $\mathrm{DBP}+++\mathrm{mm} \mathrm{Hg}$ & 87.18 & $30-163$ & 14.926 \\
\hline MBP- $\mathrm{mm} \mathrm{Hg}$ & 104.58 & $65-117$ & 17.780 \\
\hline PP-- mm Hg & 52.32 & $10-120$ & 18.029 \\
\hline
\end{tabular}

\section{Agreement}

The percentage of agreement was $40.4 \%$ (321/793) and the kappa value was 0,1 meaning slight agreement. The weighted kappa of 0,2 revealed light improvement of agreement (Table 4).

\subsection{Agreement Status and Sociodemographic Characteristics} (Table 5). There was significantly more agreement among female patients $(52.5 \%)$ versus $19.4 \%$ for male patients $(P<0.001)$. The agreement rate decreased significantly with increasing age from $87.4 \%$ for patients under 50 years to $04.9 \%$ for those older than 70 years $(P<0.001)$. 


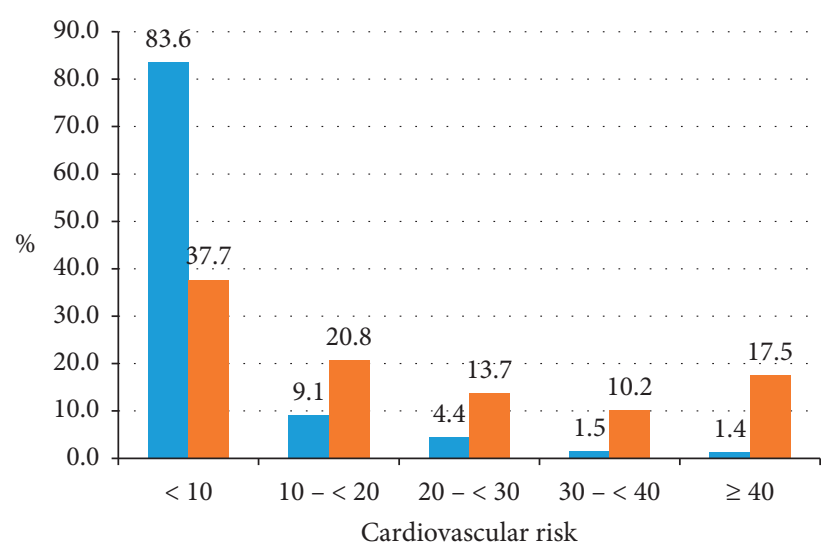

li-WHO

bmi-Frm

FIGURE 1: Body mass index-based Framingham and low-information WHO-based cardiovascular risk scores in the sample of 793 patients.

TABLE 3: bmi-based Framingham risk and low-information WHO risk prediction according to various sociodemographic characters.

\begin{tabular}{lcc}
\hline Sociodemographic characters & & $P$ value \\
\hline Medical insurance & bmi-frm & li-WHO \\
Sex & $<0.001$ & 0.792 \\
Age group (years) & $<0.001$ & $<0.001$ \\
Education level & $<0.001$ & $<0.001$ \\
Income level & 0.944 & 0.624 \\
\hline
\end{tabular}

TABle 4: Agreement between bmi-Frm and li-WHO-based prediction tool in a sample of 793 consecutive outpatients in the cardiology unit of the UH-GT.

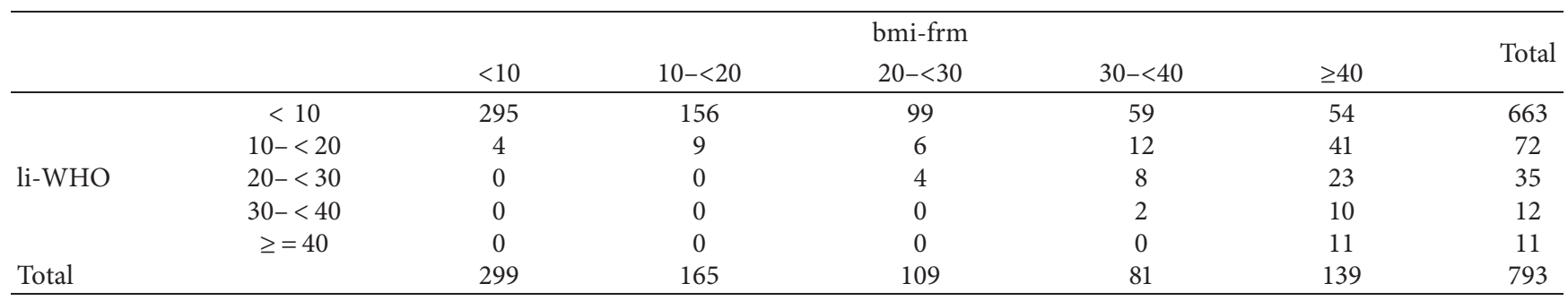

Patients without medical insurance showed more agreement with $46 \%$ versus $28.9 \%$ for those with medical insurance $(P<0.001)$.

We noticed no difference in agreement related to neither education level nor income level.

\section{Discussion}

To the best of our knowledge, this study is the first to use FRS in the Malian population in a relatively large sample of 793 outpatients seen consecutively. As lipid test is costly, we presented data for the bmi-version of the FRS [25] and the liWHO risk score.
As can be read from Figure 1, the proportion of lowrisk patients is very low compared to that of the laborless version of the WHO/ISH (Afro-D region). Clearly, the WHO/ISH prediction chart detected a few high-risk patients whereas FRS found about 10 times more high-risk subjects.

Similar African studies using WHO risk score found more low-risk patients in $87.7 \%$ for only women [26], $94.5 \%$ of slum residents [27], 76.9\% of civil servants [28], and $87.6 \%$ among adults [29].

In our study, there was no significant difference regarding education level or income level as opposed to data from other LMIC countries like Iran [22] or Tunisia [30] in 
TABLE 5: Distribution of characteristics according to agreement status of 793 patients in the cardiology unit of the UH-GT.

\begin{tabular}{|c|c|c|c|c|}
\hline \multirow{2}{*}{ Variables } & & \multicolumn{2}{|c|}{ Agreement } & \multirow{2}{*}{$P$} \\
\hline & & No (\%) & Yes $(\%)$ & \\
\hline \multirow{2}{*}{ Sex } & M & $232(80.6)$ & $056(19.4)$ & \multirow{2}{*}{$<0.001$} \\
\hline & $\mathrm{F}$ & $240(47.5)$ & $265(52.5)$ & \\
\hline \multirow{4}{*}{ Age group (years) } & $40-49$ & $035(12.6)$ & $243(87.4)$ & \multirow{4}{*}{$<0.001$} \\
\hline & $50-59$ & $121(71.6)$ & $048(28.4)$ & \\
\hline & $60-69$ & $180(88.7)$ & $023(11.3)$ & \\
\hline & $\geq 70$ & $136(95.1)$ & $007(04.9)$ & \\
\hline \multirow{4}{*}{ Education level (school years) } & None & $280(61.0)$ & $179(39.0)$ & \multirow{4}{*}{0.648} \\
\hline & Primary & $089(56.7)$ & $068(43.3)$ & \\
\hline & High school & $067(60.4)$ & $044(39.6)$ & \\
\hline & University & $036(54.5)$ & $030(45.5)$ & \\
\hline \multirow{2}{*}{ Medical insurance } & No & $290(54.0)$ & $247(46.0)$ & \multirow{2}{*}{$<0.001$} \\
\hline & Yes & $182(71.1)$ & $074(28.9)$ & \\
\hline \multirow{5}{*}{ Income level (in multiple of MOS*) } & NA & $259(59.5)$ & $176(40.5)$ & \multirow{5}{*}{0.888} \\
\hline & 1 & $057(55.9)$ & $045(44.1)$ & \\
\hline & 2 & $033(57.9)$ & $024(42.1)$ & \\
\hline & 3 & $056(62.9)$ & $033(37.1)$ & \\
\hline & 4 & $067(60.9)$ & $043(39.1)$ & \\
\hline
\end{tabular}

selected population studies with men and socially disadvantaged subjects showing a higher risk.

African data are contrasting with no difference using Framingham models for Ahmed et al. [31] and limited interchangeability of Framingham laboratory and nonlaboratory tools for Boateng et al. [32]. Both studies involved the same populations in different locations, Somalis for the first and Ghanaians for the latter.

Increasing age is known to be associated with cardiovascular risk factors (CVrf) and therefore cardiovascular events [33]. Thus, Borhanuddin et al. found a higher risk score for male subjects [34]. Female sex was also found to have a lower risk of cardiovascular events [35]. This is certainly explainable by the presence of more risk factors among males.

As per name, CVrf are associated with an increase of the global risk. This fact is known for decades in quantitative estimating by some authors [36]. It is also not surprising that the pressure values and level of risk are directly related as shown in Table 4. Advancing age has been found with increased prevalence of hypertension in Africa [37].

It is also established that anthropometric characteristics are related to cardiovascular risk. This is the case for WC $[37,38]$ and WHR [39] and explains our findings shown in Table 3.

Blood pressure is strongly related to cardiovascular risk. Several studies brought insights into the role of hypertension in the development of CVD [33, 40]. Blood pressure gradation was used in treatment [41] with our data showing a direct increase of the mean pressure values with increasing risk level.

As our study is the first of its kind, some questions remain unanswered and should be addressed in further studies.

First, this finding needs to be confirmed using a larger sample size. Some authors [42] pointed out a temporal trend in the performance of the FRS, with others recognizing the need for a model of 10-year absolute CVD risk based on a long-term cohort study for a country-based or ethnicitybased CVD.

Country-based or ethnicity-based CVD risk-prediction models are needed because of substantial variations in the four main contributors to CVD risk assessment in predictive models (mean CVD risk, mean levels of major risk factors, relative risk of a risk factor, and epidemiology of $\mathrm{CHD}$ and stroke) among different countries or ethnic groups [21]. Besides classic models with traditional CVrf, the prediction model with lifestyle factors showed an improvement of the predictive ability for cardiovascular death [43]. All these fields are to be explored based on local data.

The main problems will be certainly financial issues as large sample size studies need substantial budgets.

Female patients, those younger than 50, had significantly more agreement; this could mean that there is more agreement in patients with low risk. It is known that female patients especially before menopause had a lower risk than male patients. It is also known that the risk of cardiovascular diseases increases with age.

In this study, patients without medical insurance had a greater agreement. An explanation could be found in the proportion (not showed) of patients with medical insurance in the age groups of 50-59 and 60-69 with $35.5 \%$ and $47.3 \%$, respectively.

Education level and income level showed no relationship in this study.

\section{Limits}

Income level could not be fully studied as data for most patients were not available.

\section{Conclusion}

Using bmi-Frm and li-WHO tool gives similar risk estimation in younger female patients. Older patients must be 
evaluated using high-information tools with cholesterol, e.g., versions of the Framingham risk equation or WHO cholesterol. These data must be confirmed in further studies and compared to data from prospective studies.

\section{Data Availability}

The data used to support the findings of this study are available from the corresponding author upon request.

\section{Conflicts of Interest}

The authors declare that they have no conflicts of interest.

\section{References}

[1] Cardiovascular diseases (CVDs), 2019. https://www.who.int/ news-room/fact-sheets/detail/cardiovascular-diseases-(cvds).

[2] L. J. Laslett, P. Alagona, B. A. Clark et al., "The worldwide environment of cardiovascular disease: prevalence, diagnosis, therapy, and policy issues," Journal of the American College of Cardiology, vol. 60, no. 25, pp. S1-S49, 2012.

[3] G. A. Roth, C. Johnson, A. Abajobir, F. Abd-Allah, S. F. Abera et al., "Global, regional, and national burden of cardiovascular diseases for 10 causes, 1990 to 2015," Journal of the American College of Cardiology, vol. 70, no. 1, pp. 1-25, 2017.

[4] World Health Organization, Prevention of Cardiovascular Disease: Guidelines for Assessment and Management of Cardiovascular Risk, World Health Organization, Geneva, Switzerland, 2007.

[5] H. C. McGill, C. A. McMahan, and S. S. Gidding, "Preventing heart disease in the 21st century," Circulation, vol. 117, no. 9, pp. 1216-1227, 2008.

[6] R. L. Sacco, G. A. Roth, K. S Reddy, D. K. Arnett, R. Bonita, and T. A. Gaziano, "The heart of 25 by 25: achieving the goal of reducing global and regional premature deaths from cardiovascular diseases and stroke," Circulation, vol. 133, no. 23, pp. e674-e690, 2020.

[7] T. A. Gaziano, "Cardiovascular disease in the developing world and its cost-effective management," Circulation, vol. 112 , no. 23 , pp. 3547-3553, 2005.

[8] H. O. Bâ, I. Menta, Y. Camara et al., "Cardiovascular risk factors (CVrf) in rural and urban areas Mali: data from the step 2013 survey," World Journal of Cardiovascular Diseases, vol. 8, no. 1, pp. 35-47, 2018.

[9] A. A. Alsheikh-Ali, M. I. Omar, F. J. Raal et al., "Cardiovascular risk factor burden in Africa and the Middle East: the Africa Middle East cardiovascular epidemiological (ACE) study," PLoS One, vol. 9, no. 8, Article ID e102830, 2014.

[10] F. J. Raal, A. A. Alsheikh-Ali, M. I. Omar et al., "Cardiovascular risk factor burden in Africa and the Middle East across country income categories: a post hoc analysis of the cross-sectional Africa Middle East Cardiovascular Epidemiological (ACE) study," Arch Public Health, vol. 76, p. 15, 2018.

[11] F. P. Cappuccio and M. A. Miller, "Cardiovascular disease and hypertension in sub-Saharan Africa: burden, risk and interventions," Internal and Emergency Medicine, vol. 11, no. 3, pp. 299-305, 2016.

[12] S. Hamid, W. Groot, and M. Pavlova, "Trends in cardiovascular diseases and associated risks in sub-Saharan Africa: a review of the evidence for Ghana, Nigeria, South Africa, Sudan and Tanzania," The Aging Male, vol. 22, no. 3, pp. 169-176, 2019.
[13] D. Boateng, F. Wekesah, J. L. Browne et al., "Knowledge and awareness of and perception towards cardiovascular disease risk in sub-Saharan Africa: a systematic review," PLoS One, vol. 12, no. 12, Article ID e0189264, 2017.

[14] S. O. Oti, S. J. van de Vijver, C. Kyobutungi et al., "A community-based intervention for primary prevention of cardiovascular diseases in the slums of Nairobi: the SCALE UP study protocol for a prospective quasi-experimental community-based trial," Trials, vol. 14, no. 1, p. 409, 2013.

[15] S. Van De Vijver, S. Oti, J. Addo, A. De Graft-Aikins, and C. Agyemang, "Review of community-based interventions for prevention of cardiovascular diseases in low- and middleincome countries," Ethnicity \& Health, vol. 17, no. 6, pp. 651-676, 2012.

[16] World Health Organization, Package of Essential Noncommunicable (PEN) Disease Interventions for Primary Health Care in Low-Resource Settings, World Health Organization, Geneva, Switzerland, 2020, https://www.who.int/nmh/ publications/essential_ncd_interventions_lr_settings.pdf.

[17] M. T. Cooney, A. Dudina, R. D’Agostino, and I. M. Graham, "Cardiovascular risk-estimation systems in primary prevention," Circulation, vol. 122, no. 3, pp. 300-310, 2010.

[18] F. Farzadfar, "Cardiovascular disease risk prediction models: challenges and perspectives," Lancet Glob Health, vol. 7, no. 10, pp. e1288-e1289, 2019.

[19] WHO, WHO/ISH Cardiovascular Risk Prediction Charts, World Health Organization, Geneva, Switzerland, 2019, https://www.who.int/cardiovascular_diseases/guidelines/ Chart_predictions/en/.

[20] H. Bâ, I. Sangare, S. Camara et al., "Cardiovascular risk (CV) assessment according to the WHO cholesterol free chart in the cardiology department of university hospital Gabriel Touré," Cardiology \& Vascular Research, vol. 2, no. 2, pp. 1-5, 2018.

[21] D. Zhao, J. Liu, W. Xie, and Y. Qi, "Cardiovascular risk assessment: a global perspective," Nature Reviews Cardiology, vol. 12, no. 5, pp. 301-311, 2015.

[22] M. Nakhaie, B. Koor, S. Salehi, and F. Karimpour, "Prediction of cardiovascular disease risk using framingham risk score among office workers, Iran, 2017," Saudi Journal of Kidney Diseases and Transplantation, vol. 29, no. 3, p. 608, 2018.

[23] R. B. D’Agostino, R. S. Vasan, M. J Pencina et al., "General cardiovascular risk profile for use in primary care: the framingham heart study," Circulation, vol. 117, no. 6, pp. 743-753, 2008.

[24] J. Bosomworth, "Practical use of the Framingham risk score in primary prevention Canadian perspective," Canadian Family Physician, vol. 57, pp. 417-423, 2011.

[25] Framingham Heart Study 2019. https://www. framinghamheartstudy.org/fhs-risk-functions/ cardiovascular-disease-10-year-risk/.

[26] E. Umuerri and I. Obiebi, "Total cardiovascular risk assessment of women in delta state, Nigeria, using the World Health Organization/International Society of Hypertension risk prediction chart," Journal of Clinical and Preventive Cardiology, vol. 9, no. 1, pp. 2-7, 2020.

[27] A. Vusirikala, F. Wekesah, C. Kyobutungi, and O. Oyebode, "Assessment of cardiovascular risk in a slum population in Kenya: use of World Health Organisation/International Society of Hypertension (WHO/ISH) risk prediction charts secondary analyses of a household survey," BMJ Open, vol. 9, no. 9, p. e029304, 2019.

[28] O. A. Babatunde, S. O. Olarewaju, A. A. Adeomi et al., "10year risk for cardiovascular diseases using WHO prediction 
chart: findings from the civil servants in South-western Nigeria," BMC Cardiovasc Disord, vol. 20, p. 154, 2020.

[29] J. M. Pedro, M. Brito, and H. Barros, "Cardiovascular risk assessment in Angolan adults: a descriptive analysis from CardioBengo, a community-based survey," International Journal of Hypertension, vol. 2018, Article ID 2532345, 6 pages, 2018.

[30] O. Saidi, D. Malouche, M. O’Flaherty et al., "Assessment of cardiovascular risk in Tunisia: applying the Framingham risk score to national survey data," BMJ Open, vol. 6, no. 11, Article ID e009195, 2016.

[31] S. H. Ahmed, N. Marjerrison, M. K. R. Kjøllesdal et al., "Comparison of cardiovascular risk factors among Somalis living in Norway and somaliland," International Journal of Environmental Research and Public Health, vol. 16, no. 13, p. 2353, 2019.

[32] D. Boateng, C. Agyemang, E. Beune et al., "Cardiovascular disease risk prediction in sub-Saharan African populationscomparative analysis of risk algorithms in the RODAM study," International Journal of Cardiology, vol. 254, pp. 310-315, 2018.

[33] S. Lewington, R. Clarke, N. Qizilbash, R. Peto, and R. Collins, "Age-specific relevance of usual blood pressure to vascular mortality: a meta-analysis of individual data for one million adults in 61 prospective studies," The Lancet, vol. 360, pp. 1903-1913, 2002.

[34] B. Borhanuddin, A. Mohd Nawi, S. A. Shah et al., "10-Year cardiovascular disease risk estimation based on lipid profilebased and BMI-based framingham risk scores across multiple sociodemographic characteristics: the Malaysian cohort project," The Scientific World Journal, vol. 2018, Article ID 2979206, 8 pages, 2018.

[35] K. Velu, M. Böhm, R. Schmieder et al., "Impact of sex on cardiovascular outcome in patients at high cardiovascular risk," Circulation, vol. 126, no. 8, pp. 934-941, 2012.

[36] C. Ogedegbe, M. Joffres, M. Joffres, D. MacLean, and G. Flowerdew, "A comparative evaluation of waist circumference, waist-to-hip ratio and body mass index as indicators of cardiovascular risk factors. The Canadian Heart Health Surveys," International Journal of Obesity, vol. 25, no. 5, pp. 652-661, 2001.

[37] R. BeLue, T. A. Okoror, J. Iwelunmor et al., "An overview of cardiovascular risk factor burden in sub-Saharan African countries: a socio-cultural perspective," Globalization and Health, vol. 5, no. 1, p. 10, 2009.

[38] S. B. Van Dijk, T. Takken, E. C. Prinsen, and H. Wittink, "Different anthropometric adiposity measures and their association with cardiovascular disease risk factors: a metaanalysis," Netherlands Heart Journal, vol. 20, no. 5, pp. 208-218, 2012.

[39] M. Dalton, A. J. Cameron, P. Z. Zimmet et al., "Waist circumference, waist-hip ratio and body mass index and their correlation with cardiovascular disease risk factors in Australian adults," Journal of Internal Medicine, vol. 254, no. 6, pp. 555-563, 2003.

[40] S. S. Welborn and N. D. Wong, "Hypertension and cardiovascular disease: contributions of the framingham heart study," Global Heart, vol. 8, no. 1, pp. 49-57, 2013.

[41] P. Muntner and P. K. Whelton, "Using predicted cardiovascular disease risk in conjunction with blood pressure to guide antihypertensive medication treatment," Journal of the American College of Cardiology, vol. 69, no. 19, pp. 2446-2456, 2017.
[42] S. Rospleszcz, B. Thorand, T. De Las Heras Gala et al., "Temporal trends in cardiovascular risk factors and performance of the framingham risk score and the pooled cohort equations," Journal of Epidemiology and Community Health, vol. 73, no. 1, pp. 19-25, 2019.

[43] M. Nishimoto, M. Tagawa, M Matsui et al., "A prediction model with lifestyle in addition to previously known risk factors improves its predictive ability for cardiovascular death," Scientific Reports, vol. 9, no. 1, Article ID 12953, 2019. 